Original Research Paper

\title{
Application of Human Adipose Stem Cell (hADSC) in Collagen Gel on Rat Deep Dermal Burn
}

\author{
${ }^{1,6}$ Helsy Junaidi, ${ }^{2}$ Puji Sari, ${ }^{1}$ Dwi Pratami Septiara, ${ }^{3,4,5}$ Jeanne A. Pawitan and ${ }^{2}$ Luluk Yunaini \\ ${ }^{1}$ Master Program in Biomedical Science, Faculty of Medicine, Universitas Indonesia, Indonesia \\ ${ }^{2}$ Departemen of Biology of Medicine, Faculty of Medicine, Universitas Indonesia, Indonesia \\ ${ }^{3}$ Department of Histology, Faculty of Medicine, Universitas Indonesia, Indonesia \\ ${ }^{4}$ Stem Cell Medical Technology Integrated Service Unit, \\ Dr. Cipto Mangunkusumo General Hospital/Faculty of Medicine Universitas Indonesia, Indonesia \\ ${ }^{5}$ Stem Cell and Tissue Engineering Research Center, \\ Indonesia Medical Education and Research Institute (IMERI), Faculty of Medicine Universitas Indonesia, Indonesia \\ ${ }^{6}$ Departemen of Biology of Medicine, Faculty of Medicine, Gunadarma University
}

Article history

Received: 30-06-2019

Revised: 19-08-2019

Accepted: 12-09-2019

Corresponding Author:

Helsy Junaidi

Department of Medical

Biology, Faculty of Medicine,

Universitas Indonesia, Jl.

Salemba Raya no 6, Jakarta

10430. Indonesia

Email: dr.helsy@gmail.com

\begin{abstract}
Human adipose derived stem cells (hADSC) is expected to be an efficient solution for healing deep-dermal burns that help in wound closure. This study used 20 male Sprague dawley rats. Each rat received three wounds with different treatments (control, hADSC in collagen gel and collagen gel). Wound closure was observed at day 7th, 14th, 21th and 28th (sacrificed day) macroscopically, wound areas were measured and collagen densities were evaluated. Reduction of wound area showed significant difference between hADSC in collagen gel group and control group ( $\mathrm{p}$ 0.001 ) and between hADSC in collagen gel group and collagen gel group. The hADSC in collagen gel group had a higher collagen density compared to control and collagen gel group. Application of hADSC in bovine collagen gel in rat deep-dermal burn models provided faster and better wound healing compared with control and bovine collagen gel group.
\end{abstract}

Keywords: Burns, hADSC, PCR, Bovine Collagen Gel

\section{Introduction}

Burns are tissue damage or loss that are caused by contact with very high temperatures, chemicals, electricity, or radiation (Yefta, 2009). Complications from improper handling of burn wounds can produce scars that are tense, protruding and forming fibrotic bands that lose elasticity. The scars have different color and texture compared to the suroundig area and lead to contracture and deformity (Lawton and Dheansa, 2008). In wounds that experience spontaneous reepithelialization in less than ten days, the incidence of hypertrophic scarring and contracture is four percent. In wounds that experience spontaneous re-epithelialization are more than twenty day, hypertrophic scarring and contracture incidence increased almost twenty-fold (75$80 \%$ ). Wound management refers to wound closure procedure with surgical intervention if the wound is estimated to experience spontaneous re-epithelialization in more than twenty-one days (Yefta, 2009; 2016).

The classification of burn depth is emphasized in the potential for spontaneous re-epithelialization. Epidermal burn only involves the epidermis, so that the regeneration process of the epidermis from basal lamina is possible in five to seven days after trauma (Lu, 2015; Yefta, 2016). Superficial dermal burns involve the entire epidermal layer and the superficial dermis, limited to the dermal papilla. The dermal epidermal junction is damaged so that blisters are formed due to epidermolysis (Yefta, 2016). Appendices of the skin such as hair follicles, sweat glands, sebaceous glands are intact, so the re-epithelialization process starts at the edges of wounds and at skin appendices. The process takes place within a maximum of 14 days (Lu, 2015; Yefta, 2009; 2016).

Deep dermal burn involves damage to almost the entire dermis, with partially intact skin appendices. The re-epithelialization process lasted more than 21 days. Full Thickness Burn is characterized by damage to the epidermis, dermis and deeper tissue structures. The process of spontaneous re-epithelialization from the edges of the wound (basal membrane), as well as the skin appendix is not possible because the tissue structure is totally damaged (Yefta, 2009; 2016). 
Jacson's theory distinguishes burns into three zones, namely: Zone of coagulation, zone of stasis and zone of hyperemia (Lawton and Dheansa, 2008). The above description shows that skin damage due to burns is quite unique and interesting, it requires proper handling to yield fast wound closure, functional and aesthetic skin healing so that complications can be avoided (Ghieh et al., 2015).

The use of stem cell therapy is expected to be an efficient solution in dealing with burn problems because it is expected to help wound closure through spontaneous reepithelialization of deep burns (Morton and Phillips, 2012). Transplantation of mesenchymal stem cells is a promising cell-based therapy because they are multipotent, easily obtained and have low immunogenicity, thereby reducing rejection reaction (Schu et al., 2012).

Mesenchymal Stem Cell (MSC) is a nonhematopoietic progenitor cell that can be isolated from adult tissue and characterized in vitro by its potential to differentiate. Mesenchymal stem cells are almost present in all body tissues, thus MSCs can be isolated from bone marrow, adipose tissue, umbilical cord tissue and blood, peripheral blood, muscles, lungs, liver and dental pulp (Jin et al., 2013; Wei et al., 2013).

Human adipose tissue is rich in mesenchymal stem cell populations or commonly referred to as stem cells from human adipose tissue or commonly abbreviated as Adipose-Derived Stem Cells (ADSCs) (Zuk, 2013). ADSCs can be obtained from patient liposuction waste in beauty or plastic surgery clinics and the number of mesenchymal stem cells obtained is 400 times more than from Bone Marrow Mesenchymal Stem Cells (BMMSC) (Rodrigues et al., 2014). In vivo experiments showed that ADSCs have an ability to accelerate the process of wound healing (Im et al., 2008).

The application of ADSCs to excision wounds combined with collagen has been shown to recover wounds in experimental animals. Histological examination of wound tissue biopsy at 1 st and 2 nd week after transplantation of ADSCs showed significant tissue granulation formation, capillary formation and reepithelialization (Nambu et al., 2009).

Problems in healing burns are complex that burn wounds may end with several possibilities: Spontaneous healing, delay healing and non-healing. This study aimed to get spontaneous re-epithelialization by administering human ADSCs in collagen gel in deep burns by measuring wound closure, wound area and collagen density at several time points.

\section{Materials and Methods}

\section{Subjects}

The subjects were 20 healthy male Sprague Dawley rats with average weight 380 grams. This study had ethical approval from research ethical commitee of the Faculty of Medicine Universitas Indonesia (no. 1166/UN2.F1/ETIK/2017). This research was conducted at Animal Laboratory of Biomedic Center and Basic Technology of Health Research and Development Center of the Ministry of Health, Culture Laboratory of Medical Biology Department FKUI and Histology Laboratory of the Department of Histology Faculty of Medicine Universitas Indonesia.

\section{Deep Dermal Burn Development and Treatments}

Rats were randomly grouped into 4 groups; each group consisted of 5 rats. All rats in each group were given three deep dermal burns with a wound diameter of $12 \mathrm{~mm}$. To develop deep dermal burns, rats were anesthesized and shaved in dorsal section. An iron plate of $1.2 \mathrm{~cm}$ in diameter with a temperature of $250^{\circ} \mathrm{C}$ was pressed on shaved skin for 15 seconds (Sari et al., 2018; Yunaini et al., 2019). Rats were saccrificed on day-7, 14, -21 and -28 and burn tissues were taken for histological examinations. (Yüksel et al., 2014) Development of a deep dermal burn was proven by Hematoxyllin and eosin staining (Fig. 1).

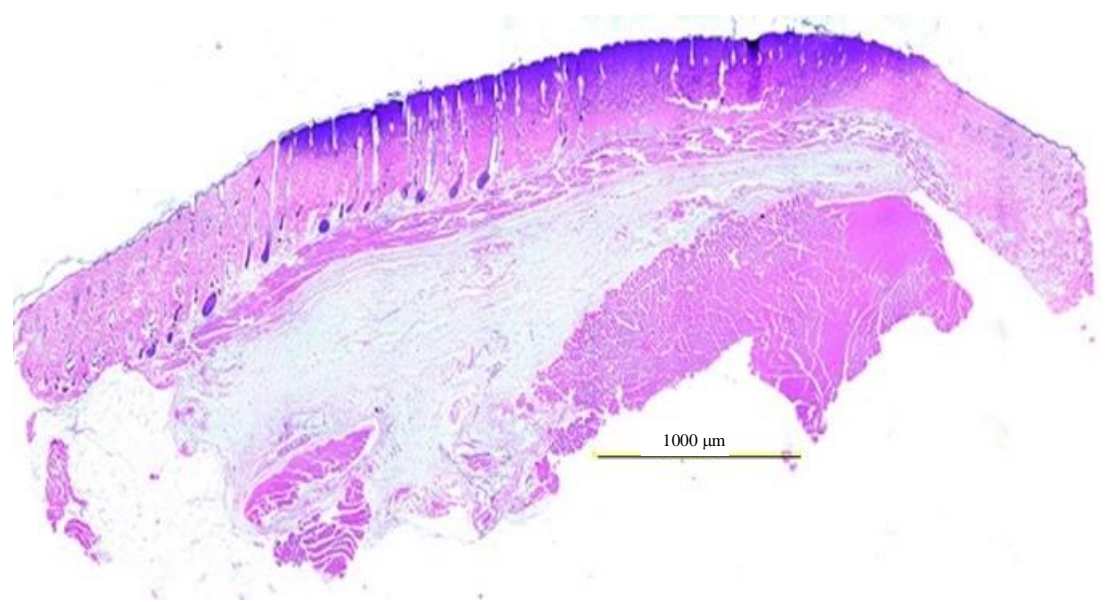

Fig. 1: Deep dermal burn of rat skin tissue stained with hematoxilyn and eosin with 40x magnification 
Isolation of Mesenchymal Stem Cells from Human Adipose Tissue

Human ADSCs were obtained according to Pawitan et al. (2016) isolation method using a coffee filter. Adipose tissues were obtained from subjects who performed liposuction at "erpour beauty clinic." The adipose tissue was filtered with a coffee filter and then washed in a mortar containing PBS until the oily material was gone (3-4x washing), the tissue looked clean and PBS solution was not greasy and had a clear color. Then the samples were digested with type 1 collagenase for $1 \mathrm{~h}$ (shaken every $5 \mathrm{~min}$ ) in a $\mathrm{CO}_{2}$ incubator at $37^{\circ} \mathrm{C}$ and $5 \% \mathrm{CO}_{2}$. Discard the top layer (oil and remnants of adipose tissue) and took the infranatant. Filter the infranatant to a $50 \mathrm{~mL}$ falcon tube using a 100 $\mu \mathrm{m}$ nylon filter. Centrifuge for $10 \mathrm{~min}, 1,200 \mathrm{rpm}$. Discard the supernatant and $10 \mathrm{~mL}$ of lysis buffer that contained $100 \mu \mathrm{L}$ fungizone and $100 \mu \mathrm{L}$ Penstrep was added to the pellet, then incubate at room temperature for $15 \mathrm{~min}$. The mixture was then centrifuged for $10 \mathrm{~min}$, $1,200 \mathrm{rpm}$. The supernatant was discarded and 1-2 mL DMEM containing 10\% fresh or expired PRP, which was processed by 1-3 freeze thaw cycles, was added to the pellet together with $1 \%$ Penstrep, $1 \%$ heparin and $1 \%$ fungizone (Suryani et al., 2013).

The pellet mixture was grown in wells of a 12 well plate with a medium of $1-1.5 \mathrm{~mL} /$ well. Twelve $\mu 1$ of the pellet mixture was use for cell counting. Cell cultures were grown in incubators at $37^{\circ} \mathrm{C}$ and $5 \%$ $\mathrm{CO}_{2}$. The cultures were observed every day. Medium change was done every 2-3 days and harvested was done after 80\% confluent (Pawitan et al., 2013; Yunaini et al., 2019). (Fig. 2).

Mesenchymal stem cell culture from human adipose tissue that was $80 \%$ confluent was harvested and characterized. The International Society of Cellular Therapy consensus stated that mesenchymal stem cells can differentiate into three main strains of mesenchymal lineages, namely osteogenic, chondrogenic and adipogenic lineage (Dominici et al., 2006).
In the differentiation stage, all cells were cultured in two wells in induction and control media. At passage two and three the stem cells were re-cultured at $37^{\circ} \mathrm{C}$ and $5 \% \mathrm{CO}_{2}$. After $50-70 \%$ confluent, one well each was induced with Stempro basal osteocyte differentiation medium supplemented with Stempro osteocyte supplement. The medium was changed every 3-4 days, after 2-3 weeks the cells would differentiate into osteocytes that was characterized by calcium deposits, which was stained by Alizarin red staining. Chondrogenic differentiation was induced by osteocyte /chondrocyte basal differentiation medium supplemented with Stempro chondrocyte supplement. The medium was changed every 3-4 days, after 2-3 weeks the cells would differentiate into chondrocytes and then characterized by Alcian blue 1\% staining. Adipogenic differentiation was induced by Stempro adipocyte basal differentiation medium supplemented with Stempro adipogenesis supplement. The medium was replaced every 3-4 days and after 2-3 weeks it would differentiate into adipogenic cells, which was characterized by lipid droplets in the cytoplasm of cells that werepositive in Oil red O staining (Schmelzer et al., 2019) (Fig. 3).

\section{Human ADSC Transplantation}

A total of $1 \times 10^{6}$ human ADSCs in collagen gel were administered on wound number $3,100 \mathrm{uL}$ collagen gel was given on wound number 2 and wound number 1 was not given any treatment. Human ADSC used passages 3 and 4 (Fig. 4).

\section{Wound Macroscopic and Microscopic Examination}

All wounds were daily observed until the sacrificial day of the rat (day-7, $-14,-21$ and -28). The measurements of macroscopis wound area were done by using digital visitrak tool (Smith and Nephew). Further, burn skin tissues were fixed in $10 \%$ buffer formalin and embedded in paraffin. Tissue samples were sectioned with microtome and were stained with Masson's Trichrome to assess collagen density.

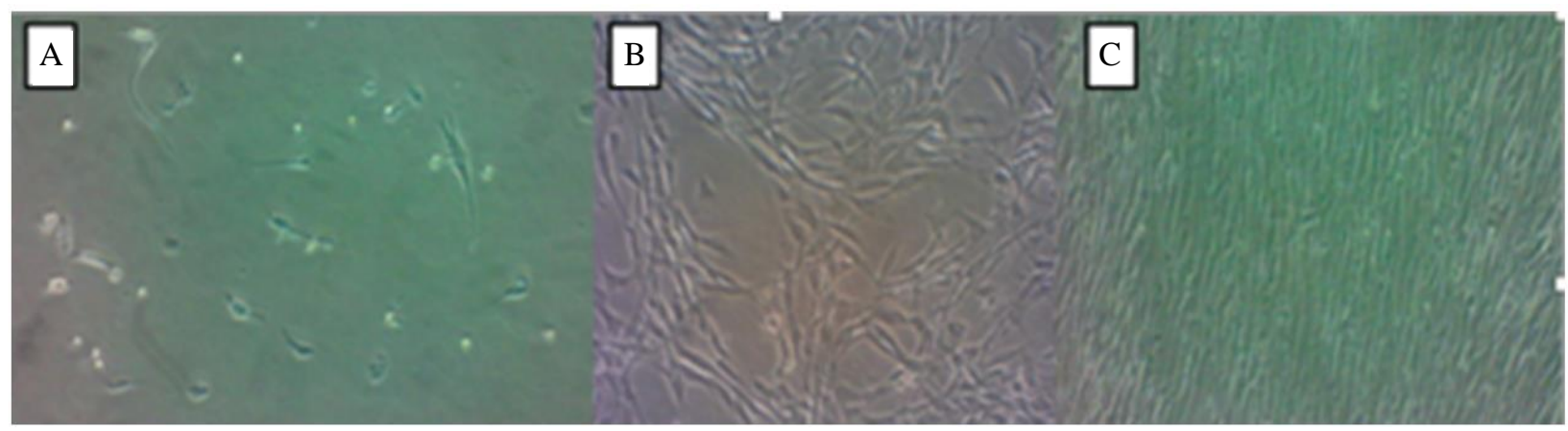

Fig. 2: Mesenchymal stem cell culture from human adipose tissue. (A) post-isolation culture. (B) Culture seven days post isolation, (C) culture 14 days post isolation. 400x magnification 


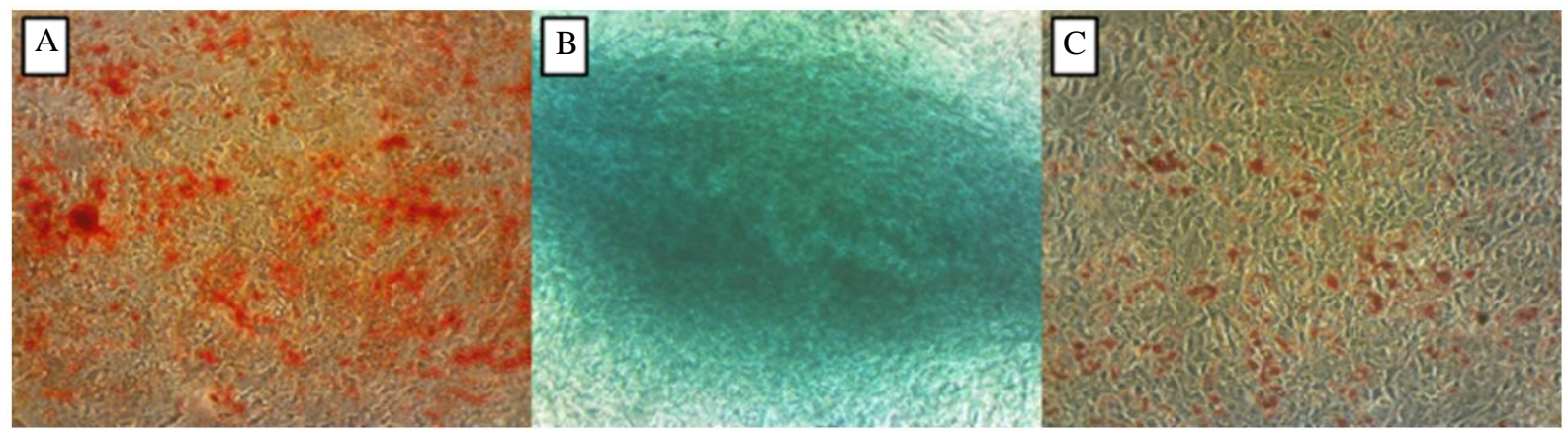

Fig. 3: Differentiation of hADSC to osteogenic cells stained by alizarin red (A) to chondrogenic cells stained by alcian blue (B) and adipogenic cells stained by red oil O (C) 400x magnification

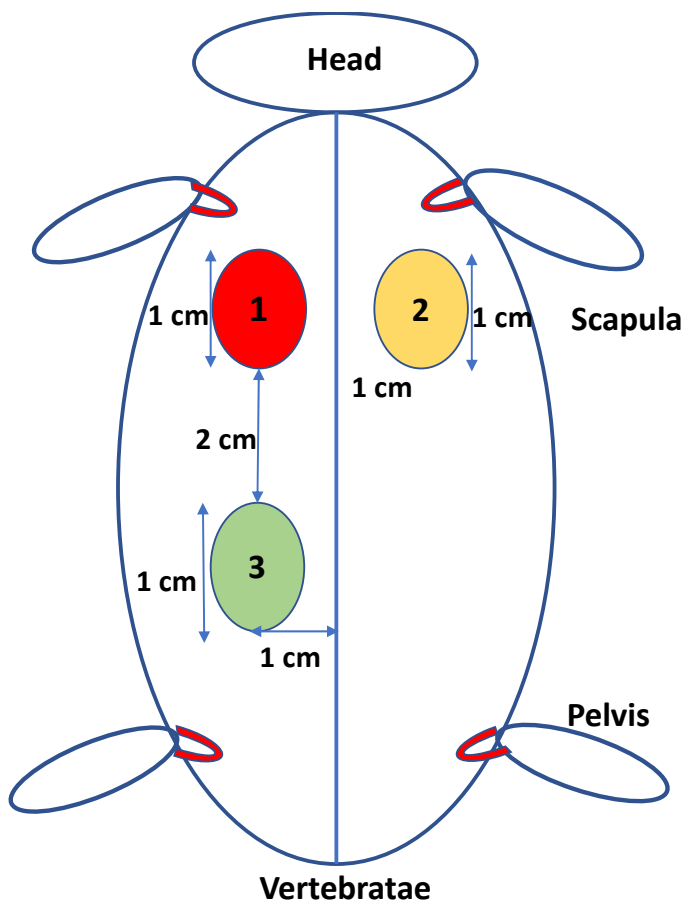

Fig. 4: Schematic picture of deep dermal burns. Each rat received three burn injuries on the dorsal section with a diameter of $1.2 \mathrm{~cm}$. 1st Wound without treatment, 2nd wound was given collagen gel and 3th wound was given human ADSCs in collagen gel (Sari et al., 2018; Yunaini et al., 2019)

\section{Masson's Trichrome Procedure}

Deparafinization was done by soaking the slides in xylol 1, xylol II, xylol III for 10 minutes. The process was continued with rehydration, in Alcohol $100 \%$, alcohol $95 \%$, alcohol $70 \%$, finally in distilled water. Then the slides were soaked in Bouin's solution at room temperature, washed in distilled water, soaked in Weigert's iron hematoxylin solution and washed in running water. Further, the slides were soaked in Phosphomolybdic acid - Phosphotungstic acid solution, followed by soaking in aniline blue, washing with distilled water and soaking in acetate water. Dehydration was done by soaking the slides in $70 \%, 95 \%$ and $100 \%$ alcohol. Further, the slides were soaked in xylol I, xylol II, xylol III, dried carefully, mounted in a glue and finally covered with a cover glass.

\section{Macroscopic Observation}

Re-epithelialization was evaluated by measuring the area of wound closure using a digital visitrak (Smith and NephewUK). The visitrak assessment sheet that was drawn on the wound slit was placed on a digital visitrak unit and the wound gap was traced using a visitrak digital stylus so that the wound area was obtained in the form of numbers in units of $\mathrm{cm}^{2}$. Macroscopic re-epithelialization was presented in the form of graphs and tables. 


\section{Collagen Density Assessment}

Collagen density was evaluated on day-7, -14, -21 and -28. Photomicrographs of the wound area were carried out using a light microscope with $40 \times 10$ magnifications using a Nikon light microscope equipped with a digital camera and using Nis Element software. Masson's Trichome staining photomicrographs were taken at five random fields from each slides and analyzed using ImageJ software (software downloaded from https://imagej.nih.gov/ij/) to determine the density of collagen. Collagen density was assessed by counting the number of pixels in the blue area. The measurement result was a number in pixels. Collagen density data was obtained by making a range from the lowest to the highest value and divided into three categories, namely strong, moderate and weak positive. The measurement results were obtained in the form of numerical and categorical data, the data was presented in the form of a range.

\section{Statistical Analysis}

Statistics were calculated with SPSS computer software for Windows (version 17.0, SPSS Inc., Chicago, IL). Wound closure area was analyzed using factorial ANOVA to compare each treatment with control and each observation day and the correlation between treatments and observation days. Collagen density was analyzed on 7th, 14th, 21st and 28th of the day. The collagen density normality test was normal ( $p$ $<0.05$ ) so statistic analysis with Chi square with collagen density categorization was done to 3 groups, i.e., low, medium, high. A probability value $(P)$ of less than 0.05 was considered to be statistically significant.

\section{Results}

Table 1: Average time (days) of wound closure based on treatment

\begin{tabular}{lllllll}
\hline & $\mathrm{N}$ & Mean & Deviation standard & \multicolumn{2}{l}{ Confident interval 95\% } & P value \\
\hline Control & 6 & 22.00 & 3.35 & 18.49 & 25.51 & 0.128 \\
hADSCs & 7 & 19.14 & 1.68 & 17.59 & 20.69 & \\
Colagen gel & 6 & 20.33 & 1.86 & 18.38 & 22.29 & 21.65 \\
Total & 19 & 20.42 & 2.55 & 19.19 & 29 \\
\hline
\end{tabular}

Table 2: Comparison of average wound closure time based on treatment

\begin{tabular}{lllll}
\hline & & Different on mean & P value & Confident interval 95\% \\
\hline Cell & Control & -2.86 & 0.046 & -5.66 \\
& Collagen Gel & -1.19 & 0.381 & -3.99 \\
\hline
\end{tabular}

Table 3: Average of wound area at day $7^{\text {th }}$

\begin{tabular}{lllll}
\hline Treatment & Mean & Deviation standard & N & P value \\
\hline Control & 1.72 & 0.15 & 20 & 0.001 \\
hADSC & 1.47 & 0.13 & 20 & \\
Bovine Colagen gel & 1.68 & 0.15 & 20 & \\
\hline
\end{tabular}

Table 4: Average of wound area at day $14^{\text {th }}$

\begin{tabular}{lllll}
\hline Treatment & Mean & Deviation standard & $\mathrm{N}$ & P value \\
\hline Control & 1.39 & 0.09 & 15 & 0.001 \\
hADSCs & 1.11 & 0.09 & 15 & \\
Collagen Gel & 1.29 & 0.09 & 15 & $\mathrm{~N}$ \\
Observation Day & Mean & Deviation standard & 15 & P value \\
7th & $1, .61$ & 0.04 & 15 & 0.001 \\
14 th & 0.92 & 0.09 & & \\
\hline
\end{tabular}

Table 5: Average of wound area in day $21^{\text {th }}$

\begin{tabular}{lllll}
\hline Treatment & Mean & Deviation standard & N & P value \\
\hline Control & 0.99 & 0.047 & 10 & 0.004 \\
hADSCs & 0.75 & 0.047 & 10 & 10 \\
Collagen Gel & 0.90 & 0.047 & $\mathrm{~N}$ & P value \\
Observation day & Mean & Deviation standard & 10 & 0.001 \\
Day 7th & 1.61 & 0.04 & 10 & 10 \\
Day 14th & 0.92 & 0.09 & 0.03 & \\
Day 21th & 0.11 & &
\end{tabular}


Table 6: Relationship between treatment and collagen density

\begin{tabular}{|c|c|c|c|c|c|c|}
\hline & & \multicolumn{4}{|c|}{ Collagen density } & \multirow[b]{2}{*}{ P Value } \\
\hline & & Low & Med & High & Total & \\
\hline \multirow[t]{6}{*}{ Treatment } & Control & 11 & 7 & 2 & 20 & 0.002 \\
\hline & & $55.0 \%$ & $35.0 \%$ & $10.0 \%$ & $100.0 \%$ & \\
\hline & hADSC + Collagen gel & 2 & 5 & 13 & 20 & \\
\hline & & $10.0 \%$ & $25.0 \%$ & $65.0 \%$ & $100.0 \%$ & \\
\hline & Collagen Gel & 7 & 8 & 5 & 20 & \\
\hline & & $35.0 \%$ & $40.0 \%$ & $25.0 \%$ & $100.0 \%$ & \\
\hline \multirow[t]{2}{*}{ Total } & & 20 & 20 & 20 & 60 & \\
\hline & & $33.3 \%$ & $33.3 \%$ & $33.3 \%$ & $100.0 \%$ & \\
\hline
\end{tabular}

Med = medium

Table 7: Collagen density on various observation days

\begin{tabular}{|c|c|c|c|c|c|c|}
\hline \multirow[b]{2}{*}{ Treatment } & & \multicolumn{4}{|c|}{ Number of animals with collagen density } & \multirow[b]{2}{*}{$\mathrm{P}$ value } \\
\hline & & Low & Medium & High & Total & \\
\hline \multirow[t]{8}{*}{ Day-7 } & & 12 & 1 & 2 & 15 & 0.01 \\
\hline & & $80.0 \%$ & $6.7 \%$ & $13.3 \%$ & $100.0 \%$ & \\
\hline & Day-14 & 2 & 9 & 4 & 15 & \\
\hline & & $13.3 \%$ & $60.0 \%$ & $26.7 \%$ & $100.0 \%$ & \\
\hline & Day-21 & 4 & 4 & 7 & 15 & \\
\hline & & $26.7 \%$ & $26.7 \%$ & $46.7 \%$ & $100.0 \%$ & \\
\hline & Day-28 & 2 & 6 & 7 & 15 & \\
\hline & & $13.3 \%$ & $40.0 \%$ & $46.7 \%$ & $100.0 \%$ & \\
\hline \multirow{2}{*}{ Total } & & 20 & 20 & 20 & 60 & \\
\hline & & $33.3 \%$ & $33.3 \%$ & $33.3 \%$ & $100.0 \%$ & \\
\hline
\end{tabular}

\section{Discussion}

\section{Observation of Macroscopic Wound Closure}

Day-7 observation showed significant differences in the average wound area between treatment groups. (Table 1) Significant difference in average wound area occurred between hADSC in collagen gel treatment group with the smallest wound area compared to control group (Tabel 3). At day-14 and -21, rats that were given hADSC in collagen gel showed the smallest wound area compared to the other treatment groups (Fig. 3 and 4) and the average wound area was significantly different between groups, where the average wound area of hADSC in collagen gel treatment group was the smallest (Table 4 and 5). Faster wound closure in hADSC in collagen gel group started from the initial stage of the wound closure process. hADSC might help wound closure through 3 methods: Cell differentiation, paracrine secretion and immunomodulation.

Previous studies revealed that ADSCs secreted various growth factors such as Keratinocyte Growth Factor (KGF), Vascular Endothelial Growth Factor (VEGF), Epidermal Growth Factor (EGF), basic Fibroblast Growth Factor (bFGF), Hepatocyte Growth Factor (HGF), TGF- $\beta$ (Atalay et al., 2014; Gimble et al., 2007; Dai et al., 2016). However, in this study there was no examination of the secretomes of the hADSC, which was the limitation of this study. Fromm-Dornieden study conducted an examination of a number of ADSC secretomes and there was a possibility that the hADSC in this study exerted the same paracrine effects that might affect wound healing process.

Skin wound healing requires integration between migration and cell proliferation, the formation of a good extracellular matrix, angiogenesis and the achievement of remodeling process (Arno et al., 2011). The use of collagen gel may provide a $3 \mathrm{D}$ structure and holds the cell to remain at the wound site so that the cell can attach and differentiate into desired cell types for wound healing, while the collagen gel treatment only may help the formation of a better extracellular matrix so that optimal migration of cells into injured tissue can occur.

The results of this study are in line with the results of a research conducted by Cheng et al. (2017) In the study, injection of ADSCs in microbead collagen scaffold was done in the wound area and the study showed that the ADSCs can proliferate and differentiate well. Further, Cheng et al. (2017) proved that stem cells that were given by local delivery method in full thickness burns with autologous keratinocyte fibrin spray showed perfect uncomplicated reepithelialization.

Wound tissue maturation was expected to show a decrease in the wound area on each observation day. Compared between the three groups, there was a significant difference in wound area between hADSC in collagen gel and both control and collagen gel treatment 
group $(\mathrm{p}=0.001)$ (Table 1$)$. This fact showed that wounds that were treated with hADSC in collagen gel had more potential in reducing wound area, because according to Dososaputro et al. (2017) hADSC can stimulate angiogenesis, epithelialization and wound healing remodeling when compared with other treatments.

This study also looked at relationship between groups of treatment with day of observation and interaction between treatments and length of day of observation. The result showed no evidence of interaction between treatment and length of day of observation. It could be interpreted that statistically the treatment of hADSC in collagen gel in deep dermal burns did not accelerate the wound healing process.

In this study perfect wound or re-epithelialization occurred before day-28 and wound healing in hADSC in collagen gel treatment was faster compared to control (Table 2). This result was consistent with the research conducted by Won-serk Kim et al. (2007) namely that wound closure in ADSC treatment was faster compared to controls.

This study used hADSC in collagen gel in rat deep dermal burn models. hADSC in collagen gel was given topically. Our results showed complete reepithelialization. This result was consistent with the research conducted by Shokrgozar et al. (2012) that showed the administration of ADSC in collagen-chitosan scaffold was able to differentiate into keratinocytes resulting in wound closure.

\section{Collagen Density}

hADSC in collagen gel treatment group showed collagen density that was higher compared to both control and collagen gel group (Table 6). This fact may happen because ADSCs release secretomes in the form of collagen I, collagen III, IV and VII (Mescher, 2013). Collagen I and III are fiber-shaped collagen, which is widely found in the skin, muscles, blood vessels and the functions are to provide resistance to strain and to maintain organ structure. Type IV collagen is found in all basement membranes and serves as a support for fine structures. Collagen type VII is present in the epithelium and tethers the basal skin lamina epithelium to the stroma below (Mescher, 2013). The presence of collagens in ADSC secretomes might cause high collagen density in hADSC in collagen gel treatment when compared with other treatments. Giving collagen gel alone showed better collagen density than control and proved that collagen was indeed needed for wound healing. However, the combination of collagen with stem cells, especially hADSC was proven to provide better collagen density. This fact supported the suggestion that collagen gel, which is bovine collagen I, could function as a 3D matrix to give strain strength to the extracellular matrix so that hADSC cells remained in the wound area and helped inflammatory cell to go to the wound area during the initial phase of wound healing. Moreover, the process of wound healing also form an extracellular matrix as a framework for cell adhesion so that cells can grow, differentiate and migrate well (Eckes et al., 2010).

Research conducted by Karimi et al. (2014) proved that administration of ADSC to full thickness burns without scaffold gave results that were not significantly different between treatment and control. Whereas the study of Shokrgozar et al. (2012) that gave ADSC with collagen-citrate scaffold showed that scaffold was effective in healing wounds and the formation of dermis and epidermis after day $14^{\text {th }}$.

In this study, collagen density was assessed to see the formation of collagen fibers by fibroblasts in the area of deep burns, which showed collagen synthesis (Fig. 7). Collagen density could also be seen from its growth trend. In the hADSC in collagen gel treatment group, (Table 7) since day-7 collagen density consistently showed higher density when compared with both control and collagen gel group (Fig. 6). This fact means that at the day-7, which was the proliferation phase, the process was characterized by proliferation and migration of fibroblasts to the wound area (Table 3). At this time fibroblasts were very active, so there was an increase in fibrogenesis followed by an increase in the amount of collagen for the formation of extracellular matrix. This increase in the amount of collagen was characterized by an increase in the density of collagen that continued to increase until day-21. This increase in collagen density occured throughout the proliferation phase because in this proliferation phase collagen deposition was still ongoing and fibrogenesis was still ongoing to form granulation tissue. Granulation tissue will form, if fibrogenesis occurs comparable to the formation of new blood vessels that later will produce re-epithelialization (Sorg, 2012).

This trend in increasing collagen density was proportional to the increase in blood vessel formation in a study conducted by Sari et al. (2018). There was an increase the amount of blood vessels until day 21th in deep dermal burns with hADSC in collagen gel treatment.

A sharp increase in collagen density on day-14 to day-21 only occurred in deep burns with hADSC in collagen gel treatment, while the collagen gel treatment and control group collagen density increase tended to be small. These facts further strengthen the possibility of hADSCs that play an important role in the formation of extracellular matrices. This opinion was supported by Kim et al. (2007) In their study they showed that ADSC promotes fibroblast proliferation because ADSC secures collagen, fibronectin and growth factors.

In the hADSC in collagen gel treatment group the observation day-28 collagen density decreased, while in the collagen gel treatment and control group collagen density tended to be the same as the previous observation day (Fig. 8). This fact might occur because the tissue had reached a balanced state. Observation on day- 28 has entered the remodeling phase of the wound healing phase. In this phase, collagen synthesis takes place simultaneously with 
the degradation of collagen. Collagenase and metalloproteinase will remove excess collagen, but new collagen synthesis continues so that collagen is more organized, fibroblast density decreases and capillary blood vessel growth also decreases. Intermolecular and intramolecular collagen cross-link results in increased wound strength (Enoch and Leaper, 2007).

The results of this study can also show that control burns that rely solely on endogenous collagen, changes in collagen density tend to be stable, as seen in Fig. 6. Burns with hADSC in collagen gel treatment and burns with collagen gel treatment, collagen density increased from the observation day-7, -14 and -21 then decreased on day28. This fact explains that at the observation of day- $7,-14$ and -21 , the collagen that was present was endogenous collagen with additional exogenous collagen. After observing the day- 21 collagen began to be degraded that was marked by a decrease in the density of collagen.

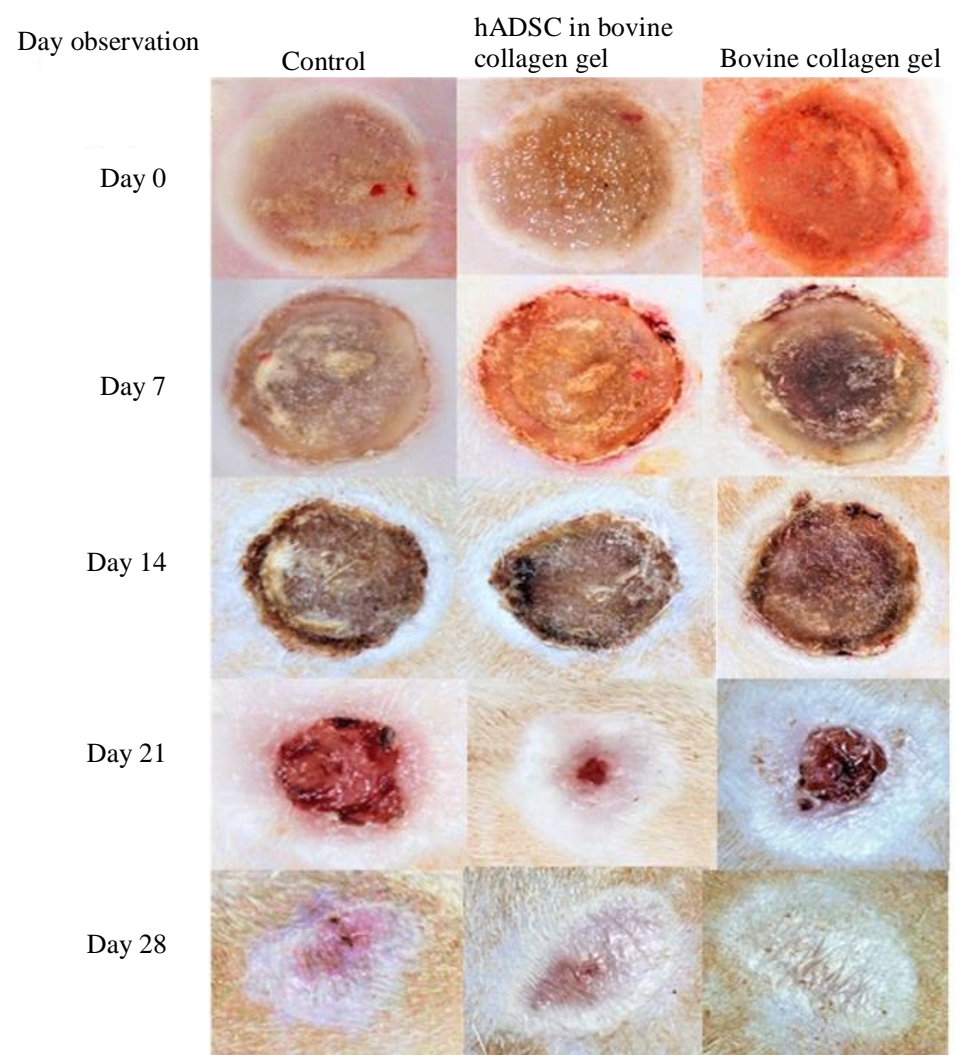

Fig. 5: Observation of wound closure process macroscopically based on observation day

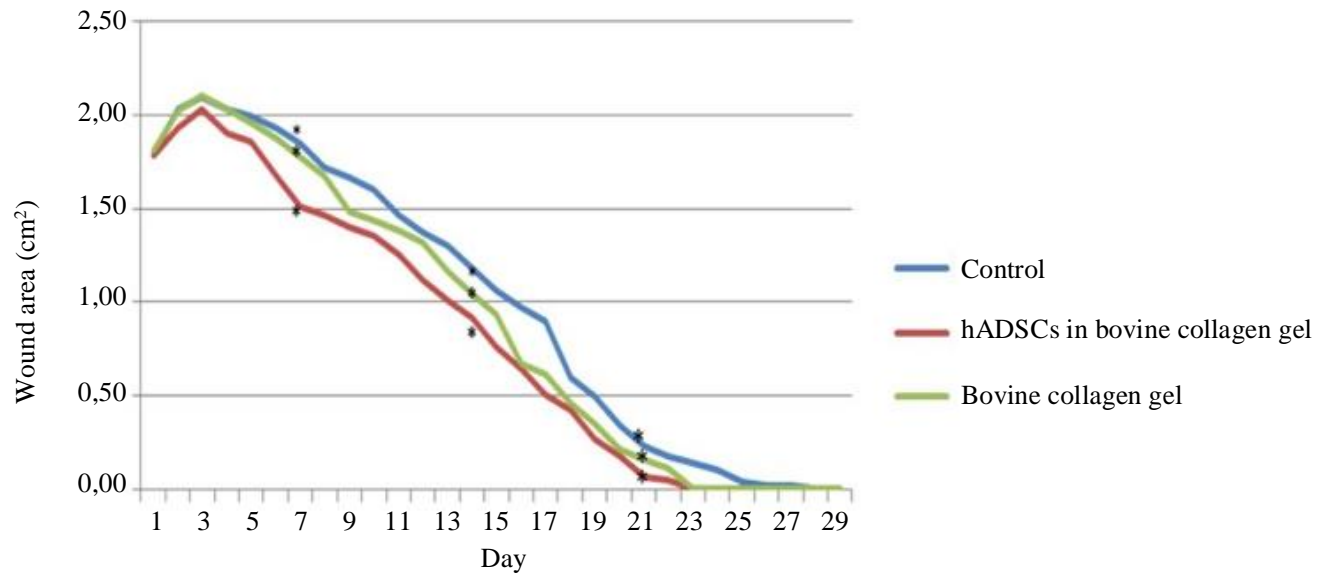

Fig. 6: Evaluation of the effect of hADSCs in collagen gel on the area of burns $\left(\mathrm{cm}^{2}\right)$. Wounds treated by hADSCs showed a smaller area of burns compared to the control and collagen group during 28 days of observation $(\mathrm{p}<0.05)$. Observation group $(\mathrm{H} 7, \mathrm{n}=$ 20, H14, $\mathrm{n}=15, \mathrm{H} 21, \mathrm{n}=10$ and $\mathrm{H} 28, \mathrm{n}=5$ ) 


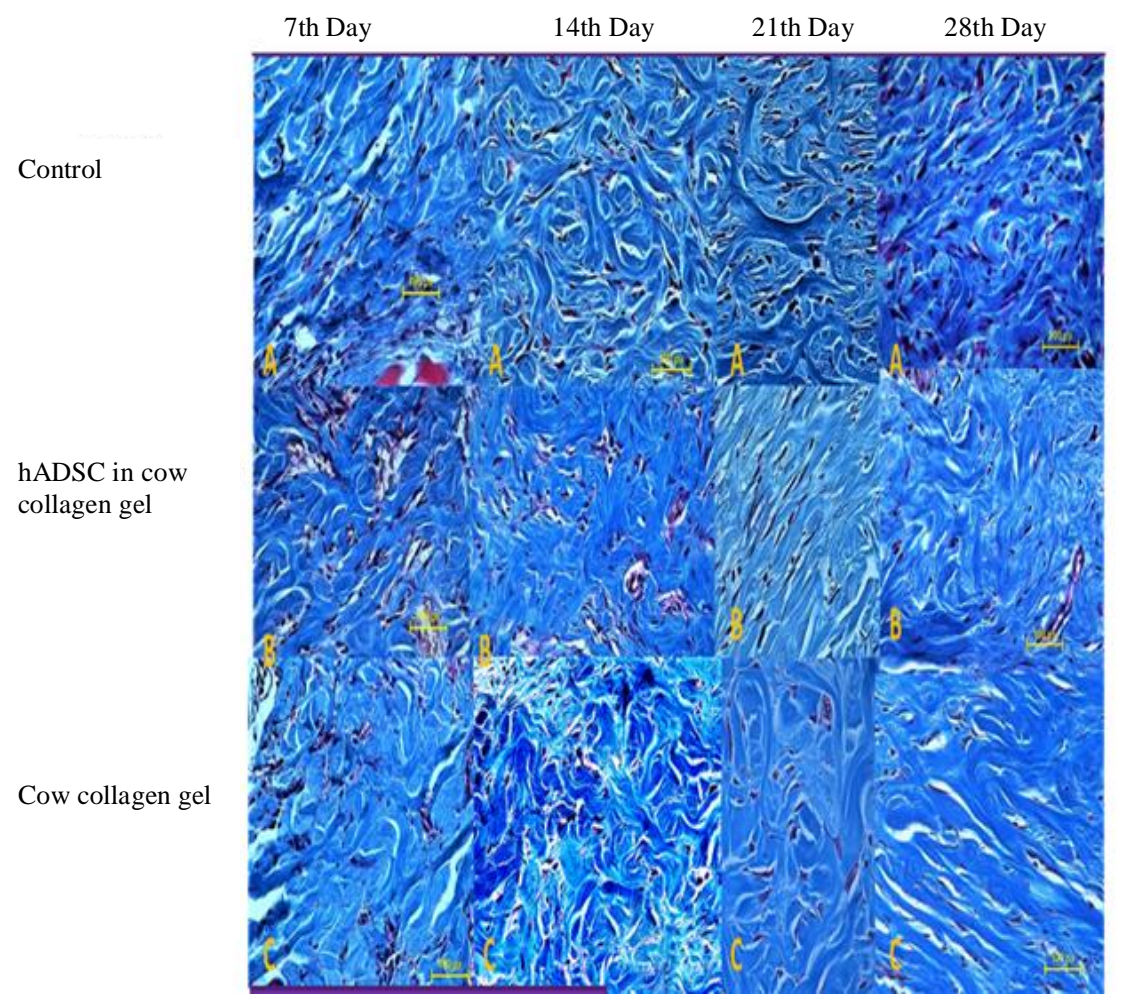

Fig. 7: Collagen with Masson trichrome staining. Wound observation on day-7, -14, -21 and -28. Control (A), hADSC in collagen gel treatment (B) and collagen gel treatment (C)

\begin{tabular}{|c|c|c|c|c|c|c|}
\hline \multirow{7}{*}{\multicolumn{2}{|c|}{ 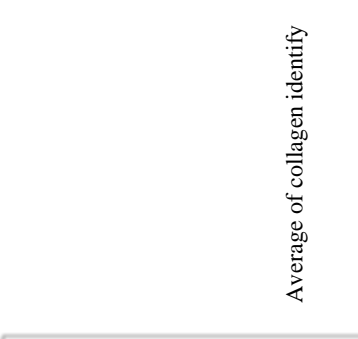 }} & $\begin{array}{l}350000,00 \\
300000,00\end{array}$ & & & 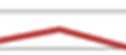 & \\
\hline & & \multirow{4}{*}{$\begin{array}{l}250000,00 \\
200000,00 \\
150000,00 \\
100000,00\end{array}$} & \multicolumn{4}{|c|}{$\mathrm{C}_{\mathrm{C}}$} \\
\hline & & & \multirow{2}{*}{\multicolumn{4}{|c|}{$\longrightarrow$}} \\
\hline & & & & & & \\
\hline & & & & & & \\
\hline & & \multirow{2}{*}{$\begin{array}{r}50000,00 \\
0,00\end{array}$} & & & & \\
\hline & & & $\mathrm{H}-7$ & $\mathrm{H}-14$ & $\mathrm{H}-21$ & $\mathrm{H}-28$ \\
\hline \multicolumn{3}{|l|}{ Control } & 167934,52 & 207068,12 & 195301,60 & 198216,84 \\
\hline \multicolumn{3}{|c|}{ — hADSC in bovine collagen gel } & 255059,80 & 263087,40 & 324722,72 & 238374,04 \\
\hline \multicolumn{3}{|l|}{ - bovine collage gel } & 174491,92 & 192344,64 & 225567,32 & 229191,04 \\
\hline & & & & Hari ke & & \\
\hline
\end{tabular}

Fig. 8: Changes in the proportion of collagen density according to observation days. The hADSC treatment group in collagen gel was higher when compared to the control and collagen gel group. Surge in collagen density in the hADSC in collagen gel treatment group on observation day- -21 . Observation day group $(\mathrm{H}-7: \mathrm{n}=5, \mathrm{H}-14: \mathrm{n}=5, \mathrm{H}-21: \mathrm{n}=5, \mathrm{H}-28: \mathrm{n}=5)$

The above results could be explained as in the early stages of wound healing fibroblasts secrete Matrix Metalloproteinases (MMP), Tissue Inhibitors of matrix Metalloproteinases (TIMP) and Glycosaminoglycans (GAG). Fibroblasts and endothelial cells began to migrate and proliferate. Endothelial cells form new capillary vessels, fibroblasts secrete collagen to form granulation tissue. Keratinocytes began to migrate from the edges of the wound along granulation tissue. Collagen gel with hADSCs and without hADSCs contributes positively to facilitate the formation of granulation tissue. This fact is supported by a study conducted by Balwada et al. (2014) who looked at the healing effects of full thickness wounds in mice with the treatment using Bovine collagen sheet with fibroblast cells. Balwada et al. (2014) showed that 
migration of cells in wound healing occurred faster and collagen made it easier for cells to attach, migrate and proliferate in the wound site.

Exogenous collagen was ideal in biomedical applications because of its biocompability and biodegradability (Chattopadhyay, 2014) As body's main structural protein, collagen is resistant to proteolysis, when endogenous MMP are formed, exogenous collagen began to be degraded so that at this stage endogenous collagen will be more dominant, which can be proven by the decrease in collagen density in this study.

Measurement results of collagen density showed that the healing quality of deep burns with hADSC in collagen gel treatment was better when compared with collagen gel treatment and control group.

\section{Conclusion}

Though human ADSCs in collagen gel in this study did not accelerate wound healing process in rats with deep dermal burns, wound area was significantly smaller compared to control or collagen gel group. Moreover, collagen density that indicated wound healing quality in rats with deep dermal burn healing was better in human ADSC in collagen gel treatment.

\section{Funding Information}

We would like to thank DRPM, which provided research funding through "Program Hibah PITTA 2016."

\section{Author Contributions}

All authors contributed equally in this work.

\section{Conflicts of Interest}

There are no conflicts of interest.

\section{Ethics}

The authors declare that this article is original and has received permission from Research Ethics Committee of Faculty of Medicine Universitas Indonesia (Approval letter No. 1166/UN2.F1/ETIK/2017).

\section{References}

Arno, A., A.H. Smith, P.H. Blit, M. Al Shehab and G.G. Gauglitz et al., 2011. Stem cell therapy: A new treatment for burns? Pharmaceuticals (Basel), 4: 1355-1380. DOI: 10.3390/ph4101355

Atalay, S., A. Coruh and K. Deniz, 2014. Stromal vascular fraction improves deep partial thickness burn wound healing. Burns, 40: 1375-1383. DOI: 10.1016/j.burns.2014.01.023
Balwada, A.K., S. Kumar, A.K. Sharma, N. Kumar and S.K. Maiti, 2014. Effect of bovine collagen sheet with fibroblast cell in full thickness skin wound healing in rat model. Res. J. Vet. Pract., 2: 91-97. DOI: $10.1517 / 14728222.10 .1 .143$

Chattopadhyay, S., 2014. Collagen-based biomaterials for wound healing.

Cheng, J.Z., A. Farrokhi, A. Ghahary and R.B. Jalili, 2017. Therapeutic use of stem cells in treatment of burn injuries. J. Burn Care Res. DOI: 10.1097/BCR.0000000000000571

Dominici, M., K. Le Blanc, I. Mueller, I. SlaperCortenbach and F. Marini et al., 2006. Minimal criteria for defining multipotent mesenchymal stromal cells. The International Society for Cellular Therapy position statement. Cytotherapy, 8: 315317. DOI: $10.1080 / 14653240600855905$

Dososaputro, I., L.T. Hoekstra, D. Rahmania and D.S. Perdanakusuma, 2017. Role of adipose-derived mesenchymal stem cells in full thickness wound healing. J. Stem Cell Res. Therapy. DOI: $10.4172 / 2157-7633.1000398$

Eckes, B., R. Nischt and T. Krieg, 2010. Cell-matrix interactions in dermal repair and scarring.

Enoch, S. and D.J. Leaper, 2007. Basic science of wound healing.

Ghieh, F., R. Jurjus, A. Ibrahim, A.G. Geagea and H. Daouk et al., 2015. Review article the use of stem cells in burn wound healing: A review. BioMed Res. Int., 2015: 2-3. DOI: 10.1155/2015/684084

Gimble, J.M., A.J. Katz and B.A. Bunnell, 2007. Adipose-derived stem cells for regenerative medicine. Circulat. Res., 100: 1249-1260. DOI: 10.1161/01.RES.0000265074.83288.09

Im, W.K., S.P. Ark, S.A. Hn, H.K. Im and J.P. Ark et al., 2008. Whitening effect of adipose-derived stem cells: A critical role of TGF-b 1. Biol. Pharm. Bull., 31: 606-610.

Jin, J.H., K.Y. Bae, M. Kim, S.J. Kwon and B.H. Jeon et al., 2013. Comparative analysis of human mesenchymal stem cells from bone marrow, adipose tissue and umbilical cord blood as sources of cell therapy. Int. J. Molecular Sci. DOI: 10.3390/ijms140917986

Karimi, H., A. Soudmand, Z. Orouji, E. Taghiabadi and S.J. Mousavi, 2014. Burn wound healing with injection of adipose-derived stem cells: A mouse model study. Ann. Burns Fire. Disasters, 27: 44-49.

Kim, W., B. Park, J. Sung and J. Yang, 2007. Wound healing effect of adipose-derived stem cells: A critical role of secretory factors on human dermal fibroblasts. J. Dermatol. Sci., 48: 15-24. DOI: $10.1016 /$ j.jdermsci.2007.05.018 
Lawton, G. and B. Dheansa, 2008. The management of major burns - a surgical perspective. Curr. Anaesthesia Critical Care, 19: 275-281.

DOI: 10.1016/j.cacc.2008.09.009

Lu, S., 2015. ChineBurn Wound Heaing. In: Chinese Burn Surgery, Zongcheng, Y. (Ed.), p: 207-207.

Mescher, A.L., 2013. Junqueira's Basic Histology. 13th Edn., Mc Graw Hill Education Medical, New York.

Morton, L.M. and T.J. Phillips, 2012. Wound healing update: Acute wound healing. Seminars Cutaneous Med. Surgery, 31: 33-37.

DOI: $10.1016 /$ j.sder.2011.11.007

Nambu, M., S. Kishimoto, S. Nakamura, H. Mizuno and S. Yanagibayashi et al., 2009. Accelerated wound healing in healing-impaired $\mathrm{db} / \mathrm{db}$ mice by autologous adipose tissue-derived stromal cells combined with atelocollagen matrix. Annals Plastic Surgery, 62: 317-321. DOI: $10.1097 /$ SAP.0b013e31817f01b6

Pawitan, J.A., I. Kurnia, D. Suryani, A. Bustami and R. Yuridian, 2016. Simple lipoaspirate washing using a coffee filter.

Pawitan, J.A., I.K. Liem, D. Suryani, A. Bustamid and R.Y. Purwoko, 2013. Simple lipoaspirate washing using a coffee filter. Asian Biomed., 7: 333-338. DOI: 10.5372/1905-7415.0703.184

Rodrigues, C., A.M. De Assis, D.J. Moura, G. Halmenschlager and J. Saffi et al., 2014. New therapy of skin repair combining adipose-derived mesenchymal stem cells with sodium carboxymethylcellulose scaffold in a pre-clinical rat model. PLoS ONE, 9: 1-10.

DOI: 10.1371/journal.pone.0096241

Dai, R., Z. Wang, R. Samanipour, K.I. Koo and K. Kim, 2016. Adipose-derived stem cells for tissue engineering and regenerative medicine applications. Stem Cells Int., 201: 19-19. DOI: $10.1155 / 2016 / 6737345$

Sari, P., D.P. Septiara, H. Junaidi, L. Yunaini and J.A. Pawitan, 2018. Effect of human adipose-derived stem cell in collagen gel on relative expression level of vascular endothelial growth factor-a of deep dermal burn healing. Asian J. Pharm. Clin. Res., 11: 384-386.

Schmelzer, E., D.T. McKeel and J.C. Gerlach, 2019. Characterization of human mesenchymal stem cells from different tissues and their membrane encasement for prospective transplantation therapies. BioMed Res. Int.

DOI: $10.1155 / 2019 / 6376271$
Schu, S., M. Nosov, L. O’Flynn, G. Shaw and O. Treacy et al., 2012. Immunogenicity of allogeneic mesenchymal stem cells. J. Cellular Molecular Med., 16: 2094-2103.

DOI: 10.1111/j.1582-4934.2011.01509.x

Shokrgozar, M.A., M. Fattahi, S. Bonakdar, I.R. Kashani and M. Majidi et al., 2012. Healing potential of mesenchymal stem cells cultured on a collagenbased scaffold for skin regeneration. Iran. Biomed. J., 16: 1-9. DOI: 10.6091/ibj.1053.2012

Sorg, J.M.R.H., 2012. Wound repair and regeneration. Eur. Surg. Res., 49: 35-43.

DOI: $10.1159 / 000339613$

Suryani, D., J.A. Pawitan, J. Lilianty, R.Y. Purwoko and I.K. Liem et al., 2013. Comparison of fetal bovine serum and platelet-rich plasma on human lipoaspirate-derived mesenchymal stem cell proliferation. Med. J. Indones. 22: 146-51. DOI: $10.13181 / \mathrm{mji} . v 22 \mathrm{i} 3.583$

Wei, X., X. Yang, Z. Han, F. Qu and L. Shao et al., 2013. Mesenchymal stem cells: a new trend for cell therapy. Acta Pharmacol. Sinica, 34: 747-754. DOI: 10.1038/aps.2013.50

Yefta, M., 2009. Luka Bakar Masalah Dan Tatalaksana. In: Luka Bakar Masalah dan Tatalaksanaluka Bakar Masalah dan Tatalaksana, Yefta, M. (Ed.), Balai Penerbit FKUI, Jakarta. pp: 1-13.

Yefta, M., 2016. Manajemen Luka Bakar. In: Luka Bakar Pediatrik, Yefta, M. (Ed.), Cv Sagung Seto, Jakarta, pp: 14-14.

Yüksel, E.B., A.M. Yıldırım, A. Bal and T. Kuloglu, 2014. The effect of different topical agents (silver sulfadiazine, povidone-iodine and sodium chloride $0.9 \%$ ) on burn injuries in rats. Plast. Surg. Int., 2014: 907082-907082. DOI: 10.1155/2014/907082

Yunaini, L., P. Sari, D.P. Septiara, H. Junaidi and R.D. Antarianto, 2019. Human adipose stem cells in collagen on angiogenesis process of burn healing in rat model: Its number of blood vessels. OnLine J. Biol. Sci., 19: 9-14. DOI: 10.3844/ojbsci.2019.9.14

Zuk, P., 2013. Adipose-derived stem cells in tissue regeneration: A review. Int. Scholarly Res. Not., 2013: e713959-e713959.

DOI: $10.1155 / 2013 / 713959$ 